Pacific Journal of Mathematics

DOMAINS OF NEGATIVITY AND APPLICATION TO
GENERALIZED CONVEXITY ON A REAL TOPOLOGICA

JACQues A. FenLAND 


\title{
DOMAINS OF NEGATIVITY AND APPLICATION TO GENERALIZED CONVEXITY ON A REAL TOPOLOGICAL VECTOR SPACE
}

\author{
JACQues A. Ferland
}

\begin{abstract}
The purpose of this paper is to derive conditions for the existence of domains of negativity, and then to determine maximal domains of convexity, quasi-convexity, and pseudoconvexity for a quadratic function defined on a real topological vector space.
\end{abstract}

1. Introduction, Martos, in [14] and [15], and Cottle and the author, in [3], [4], [6], and [7], study quasi-convex and pseudo-convex quadratic functions defined on $E^{n}$, the $n$-dimensional Euclidean space. Furthermore, in [6] and [7], the author uses the concept of domains of negativity that was introduced, mutatis mutandis, by Koecher in [11]. The purpose of this paper is to derive conditions for the existence of domains of negativity, and then to generalize the results found in [6].

In $\S 2$, we briefly review definitions needed in the rest of this paper. We also state relations between the classes of convex, quasiconvex, and pseudo-convex quadratic functions on a convex set. Conditions for the existence of domains of negativity and properties of these are given in $\S 3$. In $\S 4$, convex quadratic functions are studied. Then, domains of quasi-convexity and pseudo-convexity for quadratic forms are specified in $\S 5$, and, in $\S 6$, we extend this analysis to quadratic functions.

Note. Another approach to this theory have been used by Siegfried Schaible in "Quasi-convex Optimization in General Real Linear Spaces", Zeitschrift für Operations Research, 1972.

2. Definitions. Let $E^{1}$ denote the field of real numbers with the natural topology and let $X$ be a vector space over $E^{1}$. We assume that $X$ admits a norm, i.e., there exists a mapping $x \rightarrow|x|$ from $X$ into $E_{+}^{1}=\left\{\alpha \in E^{1} \mid \alpha \geqslant 0\right\}$ with the following properties:

(i) $|x|=0$ if and only if $x=0$,

(ii) $|\lambda x|=|\lambda||x|$ for all $\lambda \in E^{1}$ and all $x \in X$,

(iii) $|x+y| \leqslant|x|+|y|$ for all $x$ and $y$ in $X$.

A topology on $X$ is determined by this norm, and $X$, so endowed, is called a topological vector space over $E^{1}$.

Let $X$ and $Y$ be two real vector spaces. The mapping $A: X \rightarrow Y$ is a linear transformation if and only if for all vectors $x$ and $y$ in 
$X$ and for all real numbers $\alpha$ and $\beta$

$$
A(\alpha x+\beta y)=\alpha A(x)+\beta A(y) .
$$

If $Y=E^{1}$, then $A$ is said to be a linear form from $X$ into $E^{1}$.

The mapping $L: X \times X \rightarrow E^{1}$ is a bilinear form on $X$ if and only if

(i) $L(x, y)=L(y, x)$ for all $x$ and $y$ in $X$,

(ii) $L(x, y)$ is linear and continuous in $y$ for each fixed $x$. With each bilinear form $L$ is associated a unique quadratic form $Q: X \rightarrow E^{1}$ defined by

$$
Q(x)=L(x, x) \text { for all } x \in X .
$$

A quadratic function on a real vector space $X$ is a mapping $R$ : $X \rightarrow E^{1}$ defined by

$$
R(x)=1 / 2 Q(x)+P(x) \text { for all } x \in X,
$$

where $Q$ is a quadratic form and $P$ is a linear form, both defined on $X$.

The radical of a bilinear form $L$ is the set

$$
X(L)=\{x \in X \mid L(x, y)=0 \text { for all } y \in X\} \text {. }
$$

$L$ is nondegenerate on $X$ if $X(L)=0$. Otherwise, $L$ is degenerate.

If $X_{1}$ and $X_{2}$ are subsets of $X$, then the complement of $X_{2}$ relative to $X_{1}$ is the set

$$
X_{1} \backslash X_{2}=\left\{x \in X_{1} \mid x \notin X_{2}\right\}
$$

Also, the sum of $X_{1}$ and $X_{2}$ is the set

$$
X_{1}+X_{2}=\left\{x \in X \mid x=u+v, u \in X_{1} \text {, and } v \in X_{2}\right\} \text {. }
$$

If $E_{1}$ and $E_{2}$ are subspaces of $X$, then $X=E_{1} \oplus E_{2}$, the direct sum of $E_{1}$ and $E_{2}$, if and only if for each $x \in X$ there exists a unique pair $u \in E_{1}$ and $v \in E_{2}$ such that $x=u+v$.

In [11], Koecher introduces the notion of domains of positivity in a real topological vector space, and mutatis mutandis, we define a domain of negativity in $X$ determined by $L$ as a subset $Y$ of $X$ having the following properties:

(i) $Y$ is open and nonempty,

(ii) $L(x, y)<0$ for all $x$ and $y \in Y$,

(iii) for all $x \notin Y$ there exits a vector $y \in \bar{Y} \backslash X(L)$ such that $L(x, y) \geqslant 0$. (Note that $\bar{Y}$ is the closure of $Y$.)

A subset $S$ of $X$ is said to be convex if and only if for all $x, y$ in $S$ and for all $\theta \in[0,1]$

$$
x(\theta)=(1-\theta) x+\theta y \in S .
$$


Furthermore, $S$ is solid if and only if it has a nonempty interior, $S^{0}$.

The quadratic function $R(x)=1 / 2 Q(x)+P(x)$ is convex on a convex set $S$ in $X$ if and only if for all $x$ and $y$ in $S$ and for all $\theta \in[0,1]$,

$$
R((1-\theta) x+\theta y) \leqslant(1-\theta) R(x)+\theta R(y) .
$$

The quadratic function $R(x)=1 / 2 Q(x)+P(x)$ is quasi-convex on a set $S$ in $X$ if and only if for all $x$ and $y$ in $S$

$$
R(y) \leqslant R(x) \text { implies } L(x, y-x)+P(y-x) \leqslant 0 .
$$

The quadratic function $R(x)=1 / 2 Q(x)+P(x)$ is pseudo-convex on a set $S$ in $X$ if and only if for all $x$ and $y$ in $S$

$$
L(x, y-x)+P(y-x) \geqslant 0 \text { implies } R(y) \geqslant R(x) .
$$

Observe that if we take $P(x)=0$ for all $x \in X$, then (1), (2), and (3) are the conditions for the quadratic form $Q$ to convex, quasi-convex, and pseudo-convex, respectively.

If $S$ is a convex set, then denote by $C(S), Q C(S)$, and $P C(S)$ the classes of all quadratic functions $R$ that are convex on $S$, quasi-convex on $S$, and pseudo-convex on $S$, respectively.

Notice that Mangasarian's results in Chapters 6 and 9 of [13] hold for a quadratic function $R(x)=1 / 2 Q(x)+P(x)$ defined on an arbitrary real topological vector space if we replace the expression $(\nabla R(x), y-$ $x$ ) by $L(x, y-x)+P(y-x)$. (Recall that in $E^{n}$ the gradient of $R$ evaluated at $x, \nabla R(x)$, is the column vector of the partial derivatives of $R$ at $x$.) Thus, from [13, Theorem 9.1.4], we have this equivalent definition: a quadratic function $R(x)$ is quasi-convex on a convex set $S$ in $X$ if and only if for all $x, y \in S$ and for all $\theta \in[0,1]$

$$
R((1-\theta) x+\theta y) \leqslant \operatorname{Max}\{R(x), R(y)\} .
$$

Furthermore the results in [13], [Chapters 6 and 9] imply that if $S$ is a convex set in $X$, then

$$
C(S) \subset P C(S) \subset Q C(S)
$$

In [3], Cottle and the author have shown the following.

(6) Proposition. If the real valued function $h$ is quasi-convex on a nonempty convex set $S$ in $E^{n}$ and continuous on $\bar{S}$, then $h$ is quasi-convex on $\bar{S}$, the closure of $S$.

Since this result holds for a quadratic function $R$ defined on an arbitrary real topological vector space, if $S$ is convex, then

$$
Q C(S) \subset Q C(\bar{S}) .
$$


It follows from (5) and (7) that for a convex set $S \subset X$

$$
C(S) \subset P C(S) \subset Q C(S) \subset Q C(\bar{S}) .
$$

Observe the similarity with Ponstein's results for $X=E^{n}$. See [16].

3. Domains of negativity. In this section we give necessary and sufficient conditions for a bilinear form to determine a pair of domains of negativity in a real topological vector space. The importance of domains of negativity in the study of quasi-convexity and pseudoconvexity will become apparent in $\S \S 5$ and 6 .

First we introduce the following notation. For each $x \in X$ we denote by $E(x)$ the subspace generated by $x$, i.e.,

$$
E(x)=\left\{z \in X \mid z=\alpha x, \alpha \in E^{1}\right\} .
$$

Given a certain bilinear form $L$ and an arbitrary subspace $E$ of $X$, we denote

$$
E_{L}=\{z \in X \mid L(x, z)=0 \text { for all } x \in E\} .
$$

Referring to $[10, \mathrm{p} .6]$, the following is true.

(9) Proposition. If $x \in X$ and $Q(x) \neq 0$, then $X=E(x) \oplus E_{L}(x)$.

Relative to a bilinear form $L$, we say that a nonzero vector $z \in$ $X$ is

positive-valued if and only if $Q(z)>0$, negative-valued if and only if $Q(z)<0$, zero-valued if and only if $Q(x)=0$.

Suppose that $L$ is a nondegenerate bilinear form, i.e., $X(L)=0$. Furthermore, suppose there exists a vector $x \in X$. such that $Q(x)=-1$ and $E_{L}(x)$ is an inner product space where $L(u, v)$ is the inner product, i.e.,

$$
\begin{aligned}
& L(u, v)=L(v, u) \text { for all } u, v \in E_{L}(x) \\
& Q(u) \geqslant 0 \text { for all } u \in E_{L}(x) \\
& Q(u)=0 \text { implies } u=0 .
\end{aligned}
$$

For details see Schaefer [17, p. 44] or Greub [9, p. 160]. From (9),

$$
X=E(x) \oplus E_{L}(x) \text {. }
$$

Using the same type of argument as in [9, p. 268], the following can be shown. 
(10) Proposition. If $z$ is a negative-valued vector or if $z$ is a nonzero but zero-valued vector, then $L(x, z) \neq 0$.

Define the sets

$$
\begin{aligned}
& Y^{+}=\{z \in X \mid Q(z)<0 \text { and } L(x, z)<0\}, \\
& Y^{-}=\{z \in X \mid Q(z)<0 \text { and } L(x, z)>0\},
\end{aligned}
$$

Notice that $Y^{+}$and $Y^{-}$are nonempty since $x \in Y^{+}$and $-x \in Y^{-}$. It is easy to verify that

$$
\begin{aligned}
& \bar{Y}^{+}=\{z \in X \mid Q(z) \leqslant 0 \text { and } L(x, z)<0\} \cup\{0\} \\
& \bar{Y}^{-}=\{z \in X \mid Q(z) \leqslant 0 \text { and } L(x, z)>0\} \cup(0),
\end{aligned}
$$

and that $Y^{+} \cup\{0\}, Y^{-} \cup\{0\}, \bar{Y}^{+}$, and $\bar{Y}^{-}$are solid convex cones. Furthermore, a modified version of arguments [6, (3.22) and (3.32)] shows that $Y^{+}$and $Y^{-}$are domains of negativity.

The definitions of $Y^{+}$and $Y^{-}$and (10) imply the following result.

(11) Theorem. Given the pair of domains of negativity $Y^{+}$and $Y^{-}$in $X$ determined by $L$, then

(a) $z \in X^{-}=Y^{+} \cup Y^{-}$if and only if $Q(z)<0$,

(b) $z \in X^{0}=\left(\bar{Y}^{+} \backslash Y^{+}\right) \cup\left(\bar{Y}^{-} \backslash Y^{-}\right)$if and only if $Q(z)=0$,

(c) $z \in X^{+}=X \backslash\left(\bar{Y}^{+} \cup \bar{Y}^{-}\right)$if and only if $Q(z)>0$.

Since $Y^{+}$and $Y^{-}$are maximal ([11, p. 5]), then it follows from (11) that the pair $Y^{+}$and $Y^{-}$in $X$ determined by $L$ is unique.

In summary, if the vector $x \in X$ is such that $Q(x)=-1$ and $E_{L}(x)$ is an inner product space, then there exists a pair of domains of negativity in $X$ determined by $L$. This sufficient condition can be expressed into another form. To see this, we need the following result.

(12) Proposition. If there exists a vector $x \in X$ such that $Q(x)=-1$ and $E_{L}(x)$ is an inner product space, then for all $z \in X$ such that $Q(z)<$ 0 the subspace $E_{L}(z)$ is an inner product space.

Proof. For contradiction, suppose that $Q(z)<0$ for some $z \in X$ and $E_{L}(z)$ is not an inner product space. Hence, there exists a nonzero vector $y \in E_{L}(z)$ such that $Q(y) \leqq 0$. On the other hand, by definition of $x$ there exists a pair $Y^{+}$and $Y^{-}$of domains of negativity in $X$ determined by $L$.

Suppose $z \in Y^{+}$. If $Q(y)<0$, then via (11), either the pair $y$ and $z$ belongs to $Y^{+}$or the pair $-y$ and $z$ belongs to $Y^{+}$. Since $L(y, z)=$ 
$L(-y, z)=0$, in either case we have a contradiction to the definition of domains of negativity.

If $Q(y)=0$, then, via (11), either $y \in \bar{Y}^{+} \backslash Y^{+}$or $-y \in \bar{Y}^{+} \backslash Y^{+}$. Since $y \neq 0$, either the pair $z$ and $y$ or the pair $z$ and $-y$ contradicts the property that if $u \in Y^{+}$and $v \in \bar{Y}^{+} \backslash X(L)$, then $L(u, v)<0$ ([11, Theorem 1 a.]). The proof is complete.

Relying on (12), if the set $\{x \in X \mid Q(x)<0\}$ is nonempty and for each $x$ in this set the subspace $E_{L}(x)$ is an inner product space, then there exists a pair of domains of negativity. Other trivial sufficient conditions for the existence of such a pair are $Q(x)<0$ and $E_{L}(x)$ empty (i.e., $\operatorname{dim} X=1$ ). Now we turn to the necessity of these conditions.

(13) THEOREM. If there exists a pair $Y^{+}$and $Y^{-}$of domains of negativity in $X$ determined by $L$, then the set $\{x \in X \mid Q(x)<0\}$ is nonempty and for all $x \in X$ such that $Q(x)<0$ the subspace $E_{L}(x)$ is an inner product space or is empty.

Proof. Since $Y^{+}$is nonempty, it follows that $\{x \in X \mid Q(x)<0\}$ is nonempty. The second condition is shown by a similar argument as in (12), and this completes the proof.

We are left with the problem of studying conditions for the existence of domains of negativity when the bilinear form $L$ is degenerate in $X$, i.e., when $X(L) \neq 0$. Referring to Schaefer [17, p. 20], the vector space $X$ can always be expressed as

$$
X=(X / X(L)) \oplus X(L)
$$

where $X / X(L)$ is called the quotient space of $X$ over $X(L)$. It is wellknown that the bilinear form $L$ is nondegenerate on $X / X(L)$.

If there exists a pair $Y_{L}^{+}$and $Y_{\bar{L}}$ of domains of negativity in $X / X(L)$ determined by $L$, then denote

$$
\begin{aligned}
& Y^{+}=Y_{L}^{+} \oplus X(L) \\
& Y^{-}=Y_{L}^{-} \oplus X(L) .
\end{aligned}
$$

First, since $Y_{L}^{+}$and $Y_{\bar{L}}$ are nonempty and open, so are $Y^{+}$and $Y^{-}$. The other conditions for $Y^{+}$and $Y^{-}$to be domains of negativity in $X$ follow from the fact that if $x, y \in X$, then

$$
\begin{array}{ll}
x=u+t, & u \in X / X(L) \text { and } t \in X(L), \\
y=v+z, & v \in X / X(L) \text { and } z \in X(L),
\end{array}
$$

and

$$
L(x, y)=L(u, v)+L(t, z)=L(u, v) \text {. }
$$


Hence a pair $Y^{+}$and $Y^{-}$of domains of negativity in $X$ determined by $L$ exists if and only if such a pair exists when $L$ is restricted to $X / X(L)$.

4. Domains of convexity for a quadratic function. In this section, we want to determine the convex sets in $X$ over which a quadratic function is convex. In [2], Cottle has studied this problem for quadratic functions defined on $E^{n}$, and, as we shall see, these results hold on an arbitrary real topological vector space.

Using definition (1), this result follows immediately.

(14) Proposition. The quadratic function $R$ is convex on a convex set $S$ in $X$ if and only if the quadratic form $Q$ is convex on $S$.

The same kind of argument, as when the quadratic form is defined on $E^{n}$, can be used to show the following result.

(15) Proposition. The quadratic form $Q$ is convex on a convex set $S$ in $X$ if and only if for all $x$ and $y$ in $S$

$$
Q(x-y) \geqslant 0 \text {. }
$$

Notice this generalization of Cottle's result [2, (2)].

Recall that a set $K$ in $X$ is said to be a linear manifold if it is of the form

$$
K=E+x
$$

where $x \in X$ and $E$ is a vector subspace of $X$. ([1]).

With each convex set $S$ in $X$ is associated a carrying plane $K(S)$ defined as the linear manifold of least dimension which contains $S$. The same argument as in [2] shows the following property.

(16) Proposition. If the quadratic form $Q$ is convex on a convex set $S$ in $X$, then $Q$ is convex on $K(S)$.

It follows that if the quadratic form $Q$ is convex on a solid convex set $S$ in $X$, then $Q$ is convex on $X$.

5. Domains of quasi-convexity and pseudo-convexity for quadratic forms. The results found in Chapter 3 of [6] hold even for quadratic forms defined on a real topological vector space. Since only slight modifications of these arguments are needed for the generalization, we will restrict ourselves to the statements of the results. 
Suppose that $Y$ is a domain of negativity in $X$ determined by $L$.

(17) THEOREM. The quadratic form $Q$ is quasi-convex on $\bar{Y}$ and pseudo-convex on $\bar{Y} \backslash X(L)$.

(18) THEOREM. If the quadratic form $Q$ is quasi-convex, but not convex, on a solid convex set $S$, then there exists a unique pair of domains of negativity, $Y^{+}$and $Y^{-}$, in $X$ determined by $L$, and $S \subset$ $\bar{Y}^{+}$or $S \subset \bar{Y}^{-}$.

(19) THEoRem. If the quadratic form $Q$ is pseudo-convex, but not convex, on a solid convex set $S$, then there exists a unique pair of domains of negativity, $Y^{+}$and $Y^{-}$, in $X$ determined by $L$, and $S \subset$ $\bar{Y}^{+} \backslash X(L)$ or $S \subset \bar{Y}^{-} \backslash X(L)$.

Therefore, if $Y^{+}$and $Y^{-}$is a pair of domains of negativity in $X$ determined by $L$, then $\bar{Y}^{+}$and $\bar{Y}^{-}$are maximal domains of quasiconvexity, and $\bar{Y}^{+} \backslash X(L)$ and $\bar{Y}^{-} \backslash X(L)$ are maximal domains of pseudoconvexity for a quadratic form $Q$.

6. Domains of quasi-convexity and pseudo-convexity for quadratic functions.

We wish to extend the analysis of Section 5 to quadratic functions.

With each quadratic function $R(x)=1 / 2 Q(x)+P(x)$, associate the set

$$
M=\{a \in X \mid L(a, x)+P(x)=0 \text { for all } x \in X\} .
$$

A direct generalization of results in Chapter 4 of [6] gives this sufficient condition.

(20) THEOREM. If $Y \subset X$ is a domain of negativity determined by $L$ and $M$ is nonempty, then the quadratic function $R(x)$ is quasi-convex on $\bar{Y}+M$ and pseudo-convex on $\bar{Y} \backslash X(L)+M$.

Before we proceed to determine necessary conditions for the quasi-convexity of a quadratic function on a solid convex set, we have to specify under what conditions the set $M$ is nonempty.

It is obvious that the real topological vector space $X$ can be expressed as

$$
X=E^{+} \oplus E^{-} \oplus E^{0}
$$

where $E^{+}, E^{-}$and $E^{0}$ are subspaces of $X$ such that 


$$
\begin{aligned}
& Q(x)>0 \text { for all } x \in E^{+} \mid 0, \\
& Q(x)<0 \text { for all } x \in E^{-} \backslash 0 \\
& Q(x)=0 \text { for all } x \in E^{0},
\end{aligned}
$$

This decomposition may not be unique, but for the rest of this section we make the following assumption:

(21) There exists at least one decomposition

$$
X=E^{+} \oplus E^{-} \oplus E^{0}
$$

where $E^{+}$and $E^{-}$are complete (i.e., each Cauchy sequence in $E^{+}$or $E^{-}$is convergent).

Under this assumption the following is true:

(22) Proposition. If $R(x)=1 / 2 Q(x)+P(x)$, then either the set $M=\{a \in X \mid L(a, x)+P(x)=0$ for all $x \in X\}$ is nonempty or there exists a vector $t \in X$ such that $P(t) \neq 0$ and $L(x, t)=0$ for all $x \in X$.

Proof. First we show that both conditions cannot hold simultaneously. Indeed, suppose there is an $a \in M$; i.e., $L(a, x)+P(x)=$ 0 for all $x \in X$. On the other hand, if $t$ is such that $L(x, t)=0$ for all $x \in X$ and $P(t) \neq 0$, then $x=a$ gives a contradiction.

Next, suppose that if $L(x, t)=0$ for all $x \in X$, then $P(t)=0$. Hence $X=E^{+} \oplus E^{-} \oplus E^{0}$ implies that for all $x \in X$

$$
L(a, x)+P(x)=\left(L\left(a^{+}, x^{+}\right)+P\left(x^{+}\right)\right)+\left(L\left(a^{-}, x^{-}\right)+P\left(x^{-}\right)\right)
$$

where $a^{+}, x^{+} \in E^{+}$and $a^{-}, x^{-} \in E^{-}$. Relying on [17, p. 44] it follows that there exist at least one $a^{+} \in E^{+}$and one $a^{-} \in E^{-}$such that for all $x^{+} \in E^{+}$

$$
L\left(a^{+}, x^{+}\right)+P\left(x^{+}\right)=0
$$

and for all $x^{-} \in E^{-}$

$$
L\left(a^{-}, x^{-}\right)+P\left(x^{-}\right)=0 .
$$

This shows that $M$ is nonempty and the proof is complete.

Notice this proposition generalizes to an arbitrary real topological vector space $X$, satisfying assumption (21), a well-known result proved in Gale's book [8, Theorem 2.5] for the case $X=E^{n}$.

This proposition and similar arguments as in [6, (4.4), (4.13), and (4.15)] are combined to show these results.

(23) THEOREM. If the quadratic function $R(x)=1 / 2 Q(x)+P(x)$ is quasi-convex, but not convex, on a solid convex set $S$, then

(i) $M$ is not empty, 
(ii) there exists a unique pair of domains of negativity, $Y^{+}$and $Y^{-}$, in $X$ determined by $L$,

(iii) $S \subset \bar{Y}^{+}+M$ or $S \subset \bar{Y}^{-}+M$.

(24) THEOREM. If the quadratic function $R(x)=1 / 2 Q(x)+P(x)$ is pseudo-convex, but not convex, on a solid convex set $S$ in $X$, then

(i) $M$ is not empty,

(ii) there exists a unique pair of domains of negativity, $Y^{+}$and $Y^{-}$, in $X$ determined by $L$,

(iii) $S \subset\left(\bar{Y}^{+} \backslash X(L)+M\right)$ or $S \subset\left(\bar{Y}^{-} \backslash X(L)+M\right)$.

Therefore, if $M$ is nonempty and $Y^{+}$and $Y^{-}$are a pair of domains of negativity in $X$ determined by $L$, then $\bar{Y}^{+}+M$ and $\bar{Y}^{-}+M$ are maximal domains of quasi-convexity, and $\bar{Y}^{+} \backslash X(L)+M$ and $\bar{Y}-X(L)+$ $M$ are maximal domains of pseudo-convexity for a quadratic function $R$.

\section{REFERENCES}

1. C. Berge and A. Ghouila-Houri, Programming, Games and Transportation Networks, Methuen, London, 1965.

2. R. W. Cottle, On the convexity of quadratic forms over convex sets, Operations Res., 15 (1967), 170-172.

3. R. W. Cottle and J. A. Ferland, Matrix-theoretic criteria for the quasi-convexity and pseudo-convexity of quadratic functions, Linear Algebra and Its Appl., 5 (1972), 123-136.

4. - On pseudo-convex functions of nonnegative variables, Math. Programming, 1 (1971), 95-101.

5. N. Dunford and J. T. Schwartz, Linear Operators Part I and II, Interscience Publishers, Inc., New York, 1964.

6. J. A. Ferland, Quasi-convex and Pseudo-convex Functions on Solid Convex Sets, Technical Report No. 71-4, Operations Research House, Stanford University, April, 1971. 7. - Maximal domains of quasi-convexity and pseudo-convexity for quadratic functions, Math. Programming, 3 (1973), 178-192.

8. D. Gale, The Theory of Linear Economic Models, McGraw-Hill, New York, 1960.

9. W. G. Greub, Linear Algebra, Springer-Verlag, New York, 1963 (second edition).

10. I. Kaplansky, Linear Alegebra and Geometry, Allyn and Bacon, Inc., Boston, 1969.

11. M. Koecher, Positivitätsbereiche im $R^{n}$, Amer. J. Math., 79 (1957), 576-596; translated by R. W. Cottle, "Domains of Positivity in $R^{n}$ ", Technical Report No. 68-8, Operations Research House, Stanford University, May, 1968.

12. O. L. Mangasarian, Pseudo-convex functions, SIAM J. Control, 3 (1965), 281-290.

13. - Nonlinear Programming, McGraw-Hill Book Company, New York, 1969.

14. B. Martos, Subdefinite matrices and quadratic forms, SIAM J. Appl. Math., 17 (1969), 1215-1223.

15. — Quadratic programming with a quasi-convex objective function, Operations Res., 19 (1971), 87-97.

16. J. Ponstein, Seven kinds of convexity, SIAM Rev., 9 (1967), 115-119.

17. H. H. Schaefer, Topological Vector Spaces, The Macmillan Company, New York, 1966.

Received June 26, 1972. This research was supported by Canadian National Research Council, Grant No. A8312.

UNIVERSTTÉ DE MONTRÉAL 


\section{PACIFIC JOURNAL OF MATHEMATICS}

\section{EDITORS}

RICHARD ARENS (Managing Editor)

University of California

Los Angeles, California 90024

R. A. Beaumont

University of Washington

Seattle, Washington 98105
J. DUGUNDJI*

Department of Mathematics

University of Southern California

Los Angeles, California 90007

D. Gilbarg and J. Milgram

Stanford University

Stanford, California 94305

\section{ASSOCIATE EDITORS}
E. F. BECKENBACH
B. H. NeumanN
F. WOLF
K. YosHIDA

\section{SUPPORTING INSTITUTIONS}

UNIVERSITY OF BRITISH COLUMBIA
CALIFORNIA INSTITUTE OF TECHNOLOGY
UNIVERSITY OF CALIFORNIA
MONTANA STATE UNIVERSITY
UNIVERSITY OF NEVADA
NEW MEXICO STATE UNIVERSITY
OREGON STATE UNIVERSITY
UNIVERSITY OF OREGON
OSAKA UNIVERSITY

UNIVERSITY OF BRITISH COLUMBIA

UNIVERSITY OF CALIFORNIA

MONTANA STATE UNIVERSITY

UNIVERSITY OF NEVADA

OREGON STATE UNIVERSITY

OSAKA UNIVERSITY
UNIVERSITY OF SOUTHERN CALIFORNIA

STANFORD UNIVERSITY

UNIVERSITY OF TOKYO

UNIVERSITY OF UTAH

WASHINGTON STATE UNIVERSITY

UNIVERSITY OF WASHINGTON

\section{AMERICAN MATHEMATICAL SOCIETY} NAVAL WEAPONS CENTER

* C. R. DePrima California Institute of Technology, Pasadena, CA 91109, will replace J. Dugundji until August 1974. 


\section{Pacific Journal of Mathematics}

\section{Vol. 48, No. $1 \quad$ March, 1973}

Jan Aarts and David John Lutzer, Pseudo-completeness and the product of Baire

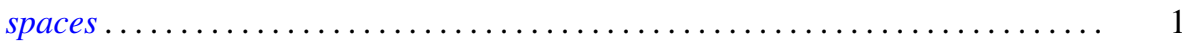

Gordon Owen Berg, Metric characterizations of Euclidean spaces ............ 11

Ajit Kaur Chilana, The space of bounded sequences with the mixed topology ..... . 29

Philip Throop Church and James Timourian, Differentiable open maps of

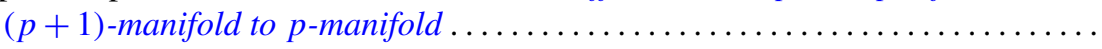

P. D. T. A. Elliott, On additive functions whose limiting distributions possess a finite

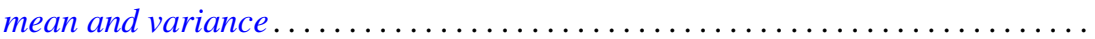

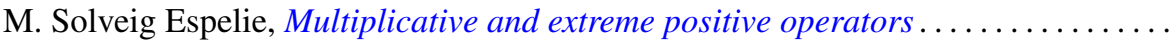

Jacques A. Ferland, Domains of negativity and application to generalized convexity

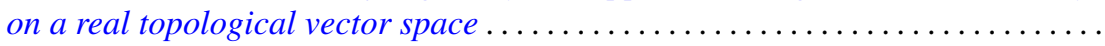

Michael Benton Freeman and Reese Harvey, A compact set that is locally holomorphically convex but not holomorphically convex ...............

Roe William Goodman, Positive-definite distributions and intertwining

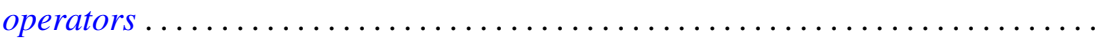

Elliot Charles Gootman, The type of some $C^{*}$ and $W^{*}$-algebras associated with

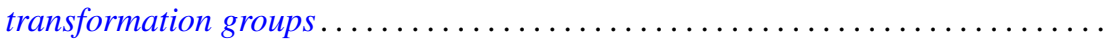

David Charles Haddad, Angular limits of locally finitely valent holomorphic

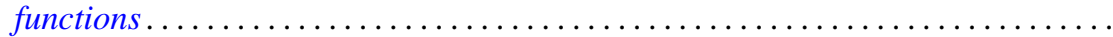

William Buhmann Johnson, On quasi-complements .

William M. Kantor, On 2-transitive collineation groups of finite projective spaces...

Joachim Lambek and Gerhard O. Michler, Completions and classical localizations of right Noetherian rings

Kenneth Lamar Lange, Borel sets of probability measures ......

David Lowell Lovelady, Product integrals for an ordinary differential equation in a Banach space

Jorge Martinez, A hom-functor for lattice-ordered groups .........

W. K. Mason, Weakly almost periodic homeomorphisms of the two sphere ....

Anthony G. Mucci, Limits for martingale-like sequences .......

Eugene Michael Norris, Relationally induced semigroups ...

Arthur E. Olson, A comparison of c-density and $k$-density ......

Donald Steven Passman, On the semisimplicity of group rings of linear groups.

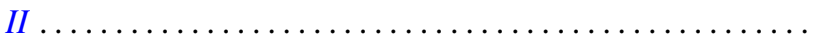

Charles Radin, Ergodicity in von Neumann algebras .

P. Rosenthal, On the singularities of the function generated by the Bergman operator of the second kind.

Arthur Argyle Sagle and J. R. Schumi, Multiplications on homogeneous spaces,

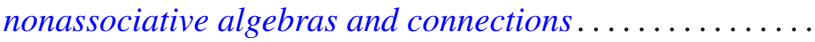

Leo Sario and Cecilia Wang, Existence of Dirichlet finite biharmonic functions on

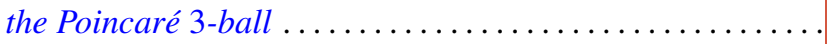

Ramachandran Subramanian, On a generalization of martingales due to Blake ..

Bui An Ton, On strongly nonlinear elliptic variational inequalities.

Seth Warner, A topological characterization of complete, discretely valued

fields. 